\title{
XXXIX. An account of some experiments on volta- electric induction
}

\section{Reuben Phillips Esq.}

To cite this article: Reuben Phillips Esq. (1848) XXXIX. An account of some experiments on volta-electric induction , Philosophical Magazine Series 3, 33:222, 260-267, DOI: 10.1080/14786444808646095

To link to this article: http://dx.doi.org/10.1080/14786444808646095

曲 Published online: 30 Apr 2009.

Submit your article to this journal

ЏII Article views: 2

Q View related articles $₫$ 
benzoic acid and glycocoll minus two equivalents of water. Before this view, however, can he considered as established, it will be necessary to examine carefully the athers derived from lecanoric, erythric and orsellic acids, in regard to their crystalline form, specific gravity, action on light, \&c., and also to compare accurately together orsellesic, erythrelesic and lecanoric acids. If the rthers derived from various sources should possess the same physical properties, and if orsellesic and erythrelesic acids should prove to be identical in properties and composition with lecanoric acid which has been dissolved in alkali and reprecipitated, then I contend that my view of the composition of these bodies will best explain the manifold changes which they undergo.

Rochdale, August 26, 1848.

XXXIX. An Account of some Experiments on Volta-Elcctric Induction. By Reuben Phillips, Esq.*

THE apparatus I employed consisted of three similar coils of covered copper wire, number 16, each coil being about 3.5 inches diameter, and containing eleven yards of wire; the ends of the wire were left exposed, so that by binding-screws the coils could be united in any order. I call these coils respectively A, B and C. To ascertain the force and direction of the induced current, I generally used an indicating helix containing a needle; this helix was made by winding covered copper wire, No. 24 , on a piece of glass tube about $\frac{1}{15}$ th of an inch in diameter; the wire was wound on the glass until it had acquired the depth of nine layers. This helix, which I call the indicator, was maintained in the same relative position with regard to an induced current, and the needles were always inserted with their points in the same relative direction; consequently the direction of the magnetism imparted by a current traversing the indicator was always the same for currents in that direction. I call a needle after it has been placed in the indicator, the indicating needle; the needles employed were of one size, and by the same maker. The magnetism imparted to them by the indicator was discovered by presenting them to one of two sewing-needles, which had been mag-

* When I had completed this paper, which I thought was altogether new, I acquainted Dr. Faraday with it, who informed me Dr. Henry had previously given the same results in a paper entitled "Contributions to Electricity and Magnetism" (Transactions of the American Philosophical Society, vol. vi.); however, Dr. Faraday thought very well of my paper and advised its publication. It is proper to remark, the paper has been a little altered since Dr. Faraday expressed this good opinion of it. 
netized and suspended so as to form a partially astatic arrangement. The battery was one on Smee's construction; it consisted of six repetitions, and the immersed portion of each plate was about 4 inches by 3.5 inches. Throughout these experiments the battery current always passed in the same direction with regard to the convolutions of the indicator.

One of the coils A was laid horizontally on a table, and the coil $B$ upon $A$; the ends of $A$ were in communication with the indicator, one end of $B$ with a wire from the battery, and the other end of $B$ dipped into some mercury into which the other galvanic pole could be plunged. A needle was now placed in the inclicator, the battery circuit completed, and the needle removed; the point of the needle was found as the marked end of a magnet.

The battery current was now sent through $B$, then the needle was placed in the indicator and the battery circuit broken, when the eye end of the needle was the marked magnetic end.

A needle was now placed in the indicator, and the battery circuit made and broken, when the eye end of the needle became as the marked end of a magnet. In these three cases the magnetism was very strong and apparently equal.

These induced currents circulate in the same direction as pointed out by Dr. Faraday, but their law of relative force is entirely different; for he found that " when the unmagnetized needle was put into the indicating helix, before contact of the inducing wire with the battery, and remained there until the contact was broken, it exhibited little or no magnetism; the first effect having been nearly neutralized by the second. The force of the induced current upon making contact was found always to exceed that of the induced current at breaking of contact*."

When the coil A was connected with a galvanometer instead of the indicator, then closing the circuit through $B$, caused the ends of the galvanometric needles to start through a space of about $\frac{1}{2}$ th of an inch, and the same on breaking the circuit; but when the end of the wire was rapidly plunged into and removed from the mercury, the needle appeared to make only an abrupt and barely perceptible motion on the circuit being completed. This agrees with the equality which Dr. Faraday and all who have followed him have found; so that although different helices give the two induced currents as various forces measured by the indicator, yet, as measured by the galvanometer, they are respectively very equal. This can only arise frum the two induced currents being about equal in

* Experimental Researches in Electricity, vol. i.p. 4. 
quantity but differing in velocity; for if a shock of electricity is sent through the wire of a galvanometer in a given time, imparting motion to the needle, then if a shock of the same quantity of electricity passes through the wire in any less time, it will impart, making up in force what it wants in time, the same motion to the needle, while it would develope a much higher magnetism in the indicator. I thus conclude, that when induced currents are produced by the discharge of a Leyden jar, the magnetized needle principally registers the variable difference of velocity between the two currents.

It will be convenient to call the current induced in the neighbouring coil by completing the battery circuit, the first induced current; and that generated while the battery current ceases, the second induced current.

It seemed to me very improbable that the first and second induced currents should travel the copper wires with such unequal velocities, and yet be exactly equal in quantity, as it would involve a strange relation between the electromotive force of the induced currents and metallic electric conduction.

By proper supports and by bending it, I arranged the wire which dipped into the mercury, so that striking the wire, as one would a key of a piano played staccato, sufficed to close and open the battery quickly whenever I pleased. The galvanometer being connected with the coil $A$ as before, the needles were made to vibrate through an arc about $\frac{1}{1}$ th of an inch long; I then struck the wire with my finger at such times as should tend to increase the motion of the needles, on the supposition that the first induced current is the more powerful; the needles were soon reduced to rest. I again made the needies vibrate, and struck the wire at those limes when so doing should add motion to the needles according to the notion that the second induced is the more powerful current, and now the needles could be kept oscillating in a small arc as long as I pleased. Therefore the second induced current is the larger quantity.

'The third coil $C$ was placed on $B$, one end of both $A$ and $C$ were united to the indicator, and the other ends bound together; $\mathrm{A}$ and $\mathrm{C}$ were so placed that on causing an electric current to circulate through $\mathrm{B}$, the current induced in $\mathrm{A}$ should act against that induced in C; I found they effectually neutralized each other.

I next placed a copper plate 4.5 inches square (which was the size of all the plates employed in these experiments) and $\frac{1}{40}$ th of an inch thick, between $B$ and $C$, and made and broke the battery circuit once through $B$, which rendered the eye end of the needle as the marked magnetic pole. 
The battery circuit was completed through $B$, and then the needle was placed in the indicator and the circuit broken, which made the eye end the marked pole.

Finally, a needle was placed in the indicator, the battery circuit closed and the needle removed; the point of the needle was the marked end. In these two last experiments the magnetism was much stronger than in the one preceding them.

'The copper plate was now removed, and a plate of zinc the $\frac{1}{100}$ dth of an inch thick was substituted for it; on'closing and opening the battery circuit once, the eye end of the needle became as the marked magnetic pole. I have thought it rather unnecessary to examine every plate as I did the copper, and I have therefore taken the most easily obtained case as a representative of the others. I should observe, too, that in all these experiments care was taken to place only unmagnetized needles in the indicator.

A plate of lead $\frac{1}{2} 0$ th of an inch thick being substituted for the zinc, produced the same effect; so also did a plate of tin of similar thickness; but when a glass plate $\frac{1}{2}$ th of an inch thick was used instead of the metal plates, I could obtain no effect on the indicating needle, although I closed and opened the battery circuit through $\mathrm{B}$ many times.

When a plate of iron $\frac{1}{20}$ th of an inch thick was placed between $B$ and $C$, the same effect as if it was a copper plate was produced on the indicating needle, but with the iron plate the induced current was evidently much more powerful.

I now proceeded to examine if a specific force belongs to each metal, or whether they all similarly effect the volta-electric induction.

Between $A$ and B I placed a plate of lead $\frac{1}{2} \overline{0}$ th of an inch thick, and between $B$ and $C$ a plate of copper of similar thickness; the battery was then closed and opened once through $B$ as before; the indicating needle had its eye end as the marked end of a magnet.

A needle was now placed in the indicator, the circuit through $B$ completed, and the needle removed; in this case the point was the marked end.

The battery was now closed through $B$, the needle placed in the indicator, and the circuit broken; this gave the eye end of the indicating needle as the marked magnetic pole. In these two last experiments the magnetism was stronger than in the experiment next going before.

When a plate of copper was substituted for the lead, no effect could be obtained in the indicator, even when this plate of copper was only $\frac{1}{x 0}$ th of an inch thick.

From the last experiment it follows that lead is much in- 
ferior to copper in cutting off the inductric infuence of a conductor; and this difference is perceptible with either the first or second induced current; also the difference of magnetizing power between the first and second induced current still remains.

Having reversed the plates, that is, having placed the copper where the lead was, and the lead where the copper was, other circunstances being the same as before, the magnetism of the indicating needle announced a current of electricity the reverse of that obtained when the plates were in their former position. In this, and generally in all the following experiments, the mercury was not employed to complete the battery circuit, which was effected by bringing together two copper wires; I have found the results identical with either method.

A plate of tin $\frac{1}{2}$ th of an inch thick and the usual size of 4.5 inches square, was placed between $A$ and $B$, and the similar plate of copper between $B$ and $C$; no effect was produced on the needle by completing the battery circuit twelve times; but when the circuit was closed and opened by drawing one of the terminal wires a few times over a file, the eye end of the needle became as the marked end of a magnet.

On placing the copper plate between $A$ and $B$, and the tin plate between $B$ and $C$, and interrupting the battery current as before, the point of the needle became as the marked end of a magnet. Hence tin acts with regard to copper as lead does, but the difference between tin and copper is less than between lead and copper.

I now placed between $A$ and $B$ a plate of zinc $\frac{1}{2}$ th of an inch thick, and between $B$ and $C$ the similar plate of copper; but although $I$ employed the file I could obtain no magnetism, even when another similar copper plate was laid on that previously used.

It occurred to me that perhaps such thick plates of metal were unsuited to develope the effects sought; for denoting by $\mathbf{P}, \mathbf{P}^{t}$ those portions of the magnetic force which found their way respectively to $A$ and $C$, then, although $\frac{P}{\mathrm{P}^{i}}$ might be very great and go on increasing with the thickness of the metal plates, yet $\mathrm{P}-\mathrm{P}^{\prime}$, which must be the magnetizing force, might be very small and capable of increase by a somewhat diminished thickness of the plates. This proved to be true.

I placed between $A$ and $B$ a plate of zinc $\frac{1}{10} 0^{2}$ th of an inch thick, and between $B$ and $C$ a plate of copper $\frac{1}{80}$ th of an inch thick; the battery circuit was closed and opened ten times, when the eye end of the indicating needle was its marked magnetic end. I now reversed the order of the plates, and closed 
and opened the circuit ten times, when the point of the needle was its marked magnetic end.

I substituted for the zinc in the two last experiments another plate of copper $\frac{1}{11} 5_{\text {th }}$ of an inch thick; in one experiment there was obtained a scarcely appreciable amount of magnetism and in the other none; in both instances the circuit was made ten times.

I could produce no effect in the indicating helix even with the aid of the file when a plate of glass $\frac{1}{2} 0$ th of an inch thick was placed on one side of $B$, and a corresponding interval filled of course with air, maintained on the other side.

I thought it interesting to ascertain what relation two similar plates of one metal, but having different thicknesses, bear to each other in limiting the action of the primary coil upon the secondary coils. I therefore placed a plate of zinc $\frac{1}{10} \mathrm{~d}$ th of an inch thick, together with a plate of glass $\frac{1}{2}$ th of an inch thick, between $\mathrm{A}$ and $\mathrm{B}$, and between $\mathrm{B}$ and $\mathrm{C}$ a plate of zinc $\frac{1}{2}$ th of an inch thick; the glass was merely employed to produce a suitable space between $\mathrm{A}$ and $\mathrm{B}$; the battery circuit was closed and opened twenty times, which made the eye end of the indicating needle to become as the marked end of a magnet. When the thin zinc and glass were placed between $B$ and $C$, and the thick zinc between $A$ and $B$, then on completing and breaking the battery circuit as before, the indicating needle showed a reverse magnetism. I made some experiments to ascertain if any difference arose by putting the glass plate on different sides of the thin metal plate, but could obtain no result to countenance such a notion.

Like results were obtained by using for the two zinc plates of the last experiments two plates of copper, one $-\frac{1}{11} 5^{\text {th }}$ of an inch and the other $\frac{1}{20}$ th of an inch thick. Nor did it appear to occasion any difference if the place of the thicker metal plate was supplied by two thinner ones.

From these experiments $I$ conclude, that a thick mass of metal plate stands to a thinner mass of the same metal as copper does to lead.

The question now arose in my mind, Does a metallic plate effect similarly, as to degree, the first and second induced currents? This was answered negatively by the following experiments.

Having removed the upper coil $C$, I united that end of the indicator, with which $\mathbf{C}$ had previously communicated, to the end of $A$ which had been joined to $C$, and placed two plates of copper, each $\frac{1}{2} \frac{1}{6}$ th of an inch thick, between $A$ and $B$; after having completed and broken the battery circuit once through $\mathrm{B}$, the point of the indicating needle was as the marked

Phil. Mag. S. 3. Vol. 33. No. 222. Oct. 1848. 
end of a magnet. One only of the copper plates was insuffcient to produce this effect.

I now used instead of the copper plates two zinc plates each $\frac{1}{20}$ th of an inch thick; the magnetism of the needle, after the completion and disruption of the battery circuit as before, was much weaker than if no plates had been interposed between the coils, and in the same direction, that is, the eye end of the needle was as the marked magnetic end. Having removed these zinc plates, and substituted a pair of zinc plates each 8 inches by 4.5 inches square, their combined thickness being 3 of an inch, and having closed and opened the battery circuit once, I found the point of the needle strongly as the marked end of a magnet; these plates had however been amalgamated and a little used for galvanic purposes. Two plates of zinc and two of tin, each $\frac{1}{20}$ th of an inch thick and of the usual size, acted the same as the two thick zinc plates in the last experiment; the tin plates alone were quite ineffective. In these experiments the induced current always passed in the directions pointed out by Dr. Faraday.

Here, then, we have the first induced current the more powerful, which is the contrary to that obtained in any of the foregoing experiments. The superiority of copper in producing this effect evidently arises from its great power of electric conduction. When a mass of glass was used instead of the copper, the eye end of the indicating needle became of course as the marked end of a magnet.

I made some experiments on the transmission of the magnetic force by the metals with the aid of two small electromagnets, the coil of one being connected with the indicator and that of the other with the battery; the poles of each were placed near to each other, so that the battery current being sent through, or checked in the coil of one magnet, induced a current in that of the other. The interposition of masses of metal between the magnetic poles occasioned no difference in the intensity of the magnetism imparted to the indicating needle when the battery circuit was closed or opened. I account for this by considering the slowness with which iron varies its magnetic state, and by supposing the action of such a metal as copper, when placed in the lines of magnetic force, retards their transmission more than air, and my apparatus not being sufficiently delicate to distinguish these two effects. Dr. Faraday first supposed such might be the action of interposed substances, and his experiments on this subject much confirm this view*.

* See Experimental Researches in Electricity, vol.i.p.545 and following. 
In concluding I cannot but observe, that the power possessed by the different metals $I$ have yet employed to shut off the circumferential influence of an electric current varies as their electric conduction; for we have them in the order of copper, zinc, tin and lead, which is that of their relative powers of electric conduction according to Harris. This view is further confirmed by the difference produced by thick and thin plates of the same metal. Indeed the following experiment clearly shows that the office of a metal, in thus intercepting voltaelectric induction, is that of an electric conductor.

The former arrangement of $\mathrm{A}, \mathrm{B}$ and $\mathrm{C}$ was restored; and of two similar plates of zinc, each $\frac{1}{10} \bar{d}$ dth of an inch thick, one was placed between $A$ and $B$ and the other between $B$ and $C$; then by the aid of a file I rapidly made and broke the battery current through $\mathrm{B}$, but could obtain no magnetism in the indicating needle. I now removed the plate from between $B$ and $\mathrm{C}$, and commencing at the middle point of one of its edges, I cut a slit at a right angle to the edge 3.5 inches long and $\cdot 1$ of an inch wide; the plate was then replaced in its former position between $\mathrm{B}$ and $\mathrm{C}$, and the battery closed and opened once through $\mathrm{B}$, which made the point of the indicating needle as the marked end of a magnet. When the position of the plates was reversed, the magnetism of the indicator was also reversed. Therefore a plate is to its fellow which has been slit as copper is to lead; and, moreover, the only assignable use of the slit is to prevent the circulation of electric currents.

With regard to constructing the three coils with which these foregoing results are to be obtained, I would remark they should not be much more than half an inch thick each; they may however contain any quantity of wire, and be of any diameter consistently with the power of the battery. I propose to call this instrument a differential volta-inductometer.

By means of my three small coils it is easy to obtain a shock from the specific induction of a metal, by removing the indicator from the ends of the coils $\mathrm{A}$ and $\mathrm{C}$, these coils being of course set so as to tend to circulate the induced current in opposite directions through each other; then having placed a plate of copper next $B$ and brought the unattached ends of $\mathbf{A}$ and $\mathrm{C}$ to one's tongue, on closing and opening the battery circuit through $\mathbf{B}$ the shock is obtained.

7 Prospect Place, Bali's Pond Road,

August $8,1848$. 